\title{
Über Melanoblasten, Hemichromasie und Faserung der Epithelzellen in breiten Condylomen.
}

Von

\author{
Prof. Dr. S. Ehrmann und Dr. Moriz Oppenheim, \\ Wien.
}

(Hiezu Taf. IX-XI.)

Wenn wir uns in der vorliegenden Abhandlung auf ein scheinbar engbegrenztes Thema der Histopathologie der Haut beschränken, so geschieht dies richt in der Absicht, nur dem Verständnisse dieses einen pathologischen Gebilde näher zu treten, sondern wir untersuchen und behandeln vielmehr in demselben ein geeignetes Paradigma für eine ganze große Reihe von Prozessen. Was wir bei dem bearbeiteten Gebilde gefunden haben, gilt bald in geringerem, bald in höherem Maße für fast alle Effloreszenzen der sekundären Syphilide und zum großen Teil auch für andere Prozesse, bej welchen die Epidermis in ihrem stratum spinosum hypertrophisch geworden ist, demnach fast für alle Akanthosen.

Ehrmann hat über Veränderungen in der Epidermis luetischer Effloreszenzen zu wiederholten Malen in verschiedenen Arbeiten gesprochen und namentlich als erster auf die $\mathrm{Hemi-}$ chromasie, sowie auf die in der Epidermis befindlichen Melanin bildenden Zellen, die Melanoblasten aufmerksam gemacht, welche hier enorm vergrößert sind. So im Jahre 1886, dann 1892, 1893 und zuletzt in der Festschrift zu Ehren von 
Prof. Pi ck im Jahre 1898. Wenn hier wiederum der Gegenstand aufgenommen wird, so geschieht es nur deshalb, weil mittlerweile neue Gesichtspunkte in die Diskussion eingeführt wurden, von denen aus die Arbeit neu in Angriff genommen werden mußte.

In erster Linie bezieht sich dies auf die Frage der Epithelfaserung und ihre Beziehung zu den Melanoblasten.

Die Epithelfaserung wurde zwar auch von dem Genannten bereits in einer Publication und in einer Demonstration der Wiener dermatologischen Gesellschaft gestreift und in einem metho. dischen Artikel im Archiv für mikroskopische Anatomie behandelt, äußere Umstände hatten aber die fortgesetzte Bearbeitung des Themas von dessen Seite verhindert. Zu diesen Hindernissen gehörten nicht in allerletzter Linie die Intoleranz desselben gegen Xyloldämpfe, welche es demselben unmöglich machten, die Methode der Epithelfärbung weiter anzuwenden; er begrüßte es daher mit Freuden, daß der Jüngere ihm die Hand zur Mitarbeit geboten hat.

Die Arbeit selbst bezieht sich zum Teil auf ältere Präparate und in Vorrat gehaltene Abbildungen, zum Teil auf neu angefertigte Präparate und deren Bilder. Die Objekte, aus denen diese Präparate angefertigt wurden, waren breite Kondylome. Sie stammten ron den kleinen und großen, stark pigmentierten Labien brïnetter Personen, waren erbsenbis haselnußgroß und wurden unmittelbar nach der Excision in absolutem Alkohol fixiert, dann in Alkohol gehärtet und in Paraffin eingebettet. Die Schnittdicke variierte von $3-6 \mu$, so daß diese wichtige Forderung der Kromayerschen Modifikation der Weigertschen Fibrinfärbungsmethode zur Darstellung der Epithelfasern durchgehends erfüllt war. Es kann also gegen uns nicht der Vorwurf erhoben werden, daß wir Trugbilder vor uns hatten, da genügend dünne Schnitte angefertigt wurden, wie übrigens auch aus den Abbildungen $\mathrm{zu}$ ersehen ist.

Von Färbungsmethoden wurden außer der Kromayerschen Modifikation der W e ig er ts c h e n Fibrinfärbungsmethode die Methode von Unna (Färbung mit polychromem Methylenblau durch 2 Minuten, Auswaschen in angesäuertem Wasser, Be- 
handlung mit $1 \%$ frisch bereiteter Ferricyankaliumlösung Fig. 9 wurde so angetertigt), sowie die Herxheimersche Methode mit Cresylechtviolett und Essigäther angewendet. Die beiden letzteren Methoden geben zwar lange" nicht so scharfe Bilder wie die ersteren, doch zeigen sie auch viel weniger Schrumpfungserscheinungen und Kunstprodukte. Klare Bilder über das Verhältnis der Epithelfasern zum Pigmente erhält man nur durch die Kromayersche Methode, daher wurde diese vorzugsweise gebraucht. Nachdem wir das breite Kondylom untersucht hatten, wandten wir unsere Aufmerksamkeit auch anderen normalen und pathologischen Hautpartien zu. So untersuchten wir pigmentierte $\mathrm{Nae}$ i, Epheliden, Negerhaut, Kopfhaut, Ochsenconjunctiva, Ochsenmaul, Conjunctiva rom Rindsembryo, Variola etc. Von allen diesen Objekten lieferte aus weiter unten angegebenen Gründen die nässende Papel die schönsten Bilder sowohl bezüglich der Melanoblasten als auch der Hemichromasie; wir beziehen uns daher vorzugsweise, wie auch der Titel sagt, auf Präparate vom breiten Kondylom.

Die an der Grenze der Epidermis, sowie in dieser selbst befindlichen Melanoblasten werden, wie $\mathrm{Ehrmann}$ an verschiedenen Stellen angeführt hat, ähnlich wie die Blutgefäßkapillaren an die Epithelzellen selbst durch den syphilitischen Prozeß hypertrophisch, um dann zu Grunde zu gehen. Es geht ihnen ebenso wie den Melanoblasten der Cutis, mit denen sie in genetischem Zusammenhang stehen.

Früher haben die Epithelzellen ihr Pigment dadurch verloren, daß dasselbe allmählich in die Hornschichte geschoben wurde, während die allmählich nachwachsenden Epithelzellen pigmentärmer und schließlich pigmentlos nachwachsen, weil sie durch die Veränderungen, welche bei Akanthose im Protoplasma vor sich gehen, die Fähigkeit verloren haben, Melanin aus den Melanoblasten aufzunehmen. Diejenigen Epithelzellen, die noch von frïher Pigment besitzen, behalten zwar dieses, nehmen aber kein neues mehr anf. Dies geschieht in einer Zone, welche etwas nach innen von der äußersten Randzone des breiten Kondyloms liegt, wo die Melanoblasten der Epidermis und Cutis noch vorhanden, ja sogar vergrößert und 
vermehrt sind, während in der Randzone selbst die Epithelzellen noch viel Pigment enthalten, aber bereits ein Aufsteigen desselben erkennen lassen.

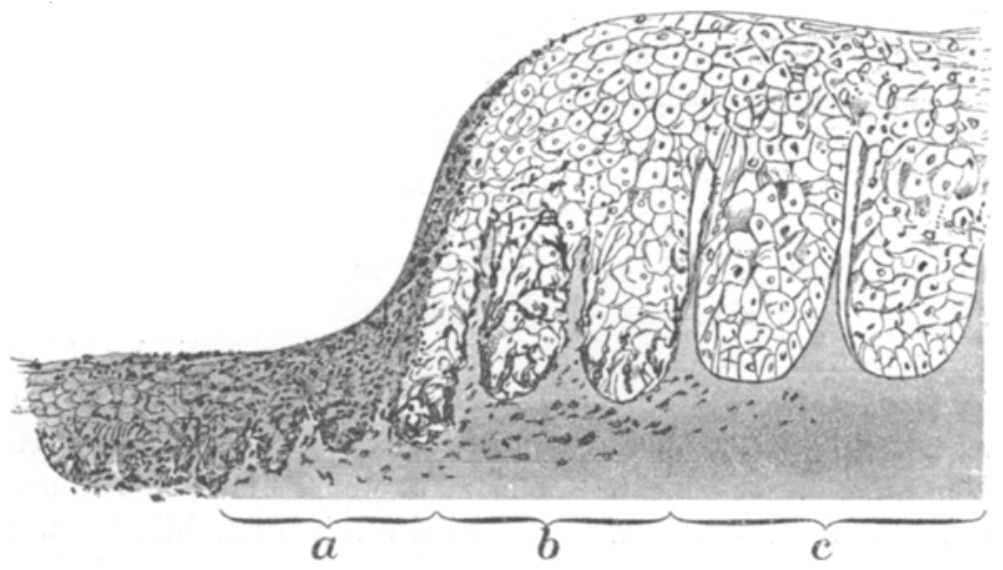

In der äußersten Randzone haben, wie schon früher beschrieben wurde, noch alle Epithelzellen ihr Pigment (Zone a der Textfigur).

In der zunächst gelegenen Zone haben die untersten Epithelzellen nur mehr wenig oder gar kein Pigment, dagegen sind die oberen Epidermisschichten noch pigmentiert. Während die unteren Schichten immer mehr und mehr pigmentlos werden, zieht sich die Pigmentierung auf immer höhere Schichten zurück, bis man schließlich nur Pigment in der Hornschicht nachweisen kann, das schließlich mit ihr abgestoßen wird. Dabei liegen noch vergrößerte Melanoblasten zwischen den akanthotik ch vergrößerten Epithelzellen (Zone $b$ ).

Im Zentrum der Papel sind die Epithelzellen ganz pigmentlos; es fehlen aber auch die Melanoblasten sowohl in der Epidermis als Cutis (Zone c).

In der Zone $b$ sind nun äußerst günstige Verhältnisse für das Studium der Epithelfaserung und der Melanoblasten, weil man letztere von den pigmentlosen Epidermiszellen sehr gut scheiden kann, wodurch Irrtümer, wie solche in der Literatur selbst gewiegten Mikroskopikern widerfahren sind, nicht leicht zu stande kommen können. Von großem Vorteil für 
die objektive Beobachtung ist auch hier die größere Weite der intercellulären Räume.

Wir wollen zunächst die Veränderungen, wie sie an den Epithelzellen nachgewiesen werden, beschreiben.

Sie erscheinen in allen Schichten des Stratum Malpighi vergrößert sowohl in der Keimschicht, als auch im Stratum spinosum und granulosum. Ihre Anzahl ist aber anch gegenüber der Norm zweifellos vermehrt, wie man aus den gerade hier besonders reichlich und schön ausgebildeten Mitosen erkennen kann. Dadurch werden die Leisten des Rete malpighi breiter, oft auch länger, die Papillen dagegen eingeengt.

Dies ist jedoch nicht bei allen Kondylomen gleichmäßig zutreffend; es hängt von der Gestaltung der Verhältnisse in der Cutis ab, auf die Ehrmann in einer anderen Arbeit näher eingehen wird. Das Kondyloma latum istein geradezu vorzügliches Objekt zur Darstellung der Verhältnisse der Epithelfasern, ähnlich wie andere mit Akanthose einhergehende Hautveränderungen.

In der Basalzellenschicht und den unmittelbar auf diese folgenden Zellenlagen haben die Fasern im allgemeinen einen zur Basallinie d. h. zur Grenzlinie zwischen Epithel und Cutis senkrechten Verlauf; also größtenteils parallel mit der Längsachse der cylindrischen Basalzellen. In den mittleren Schichten des Stratum spinosum ändert sich die Richtung insoferne, als es nicht möglich ist eine Hauptverlaufsrichtung der Fasern zu konstatieren. In dieser Schichte finden sich auch jene sternförmigen Epithelzellen K romayers, die dadurch zu stande kommen, daß eben ihre Epithelfasern nach allen Richtungen des Raumes mehr odter weniger zu Bündeln angeordnet verlaufen. Sowie wir uns dem Stratum granulosum nähern und die Epithelzellen flach werden oder in eine zur Hautoberfläche parallele Lage übergehen, nehmen auch die Epithelfasern eine derartige Richtung an. Sie verlaufen senkrecht zu den Basalepithelfasern, mehr oder weniger parallel mit der Hautoberfläche, sie haben eine Drehung um $90^{\circ}$ gemacht. Die Fasern zeigen eine verschiedene Dicke; die in der peripheren Zone des Zellprotoplasmas verlaufenden sind scheinbar die dicksten, sie geben den Kontur der Zelle an. Je näher sie dem Kerne kommen, desto dünner werden sie dem Anscheine nach; auch die Interspinalfasern sind scheinbar dünner. Was die Form der Fasern betrifft, so sind sie meistens gerade oder sanft S-förmig geschwungen, parallel, fein wellig, so daß sie den Eiudruck dünner Seidenfäden, die man aus einem Seidengewebe gezogen hat, machen. Die Randfasern der Basalzellen sind sebr dick und sehr häufig spiralig gewunden, wie schon wiederholt beschrieben wurde. Nirgends läßt sich in dieser Schicht ein direkter Zusammenhang $z$ wischen Bindegewebs- und Epithelfasern feststellen. Die letzteren gehen zwar eine kurze Strecke in das Bindegewebe hinein, zeigen aber niemals direkt als Fortsetzung eine Bindegewebsfaser. Im Gegenteil, an manchen Stellen sehen sie geradezu wie abgeschnitten aus. 
Im Stratum granulosum sind die Fasern, wenn sie gefärbt erscheinen, ebenso deutlich wie in anderen Schichten, in der Hornschicht, wo dieselben erhalten waren, haben wir sie nie gefärbt gesehen.

Was den feineren Bau des Zellprotoplasmas betrifft, so konnten wir an unseren Präparaten folgende Tatsachen konstatieren: An den Präparaten, die nach Kromayer behandelt worden sind, kann man in der Tat sehen, daß das Protoplasma der Epithelzellen scheinbar aufgelöst erscheint in ein Strickwerk von Fasern, welche wie ein komplizierter Korb den Kern umgeben. Zu gleicher Zeit aber sieht man, daß der Kern in einer Höhle liegt, welche offenbar erst durch die Präparation gebildet wurde, teils durch Retraktion der Fasermasse vom Kern, teils durch Formveränderungen des Kernes von verschiedener Intensität. Manchmal hat sich der Kern nur wenig vom umgebenden Gerüstwerk zurückgezogen, ein andermal und das ist in jenen Fällen, wo das Gerüstwerk am deutlichsten ist, hat er mannigfache Formveränderungen erlitten und ist von verschiedenen Seiten eingedrückt.

In vielen Fällen erscheint er an den Rand dieser Höhle zu einem schalenförmigen auf dem Durchschnitt halbmondförmigen Gebilde beiseite gedrängt. Die Fasern, welche den Kern umgeben, scheinen sich nach außen als die spinalen Fortsätze von Epithelzelle zu Epithelzelle fortzusetzen.

Nichts destoweniger bleibt das Bild der Zelle erhalten und man kann sich in allen Fällen, wo der Schnitt durch die Zellenmitte und nicht bloß durch die Peripherie derselben geführt ist, überzengen, daß zu jedem Kerne das dazu gehörige Fasersystem gehört und daß zwei benachbarte solche Systeme ganz deutlich durch den interspinalen Raum voneinander geschieden sind, der nur durch kurze radiär gestellte, immer als solche erkennbare Spinalfortsätze überbrückt erscheint. Nur im Stratum germinativum ist die Lagerung der Epithelfasern scheinbar etwas anders. Die Fasern verlaufen hier mehr senkrecht zur Epithelbasis, die interspinalen Räume erscheinen schmäler, oft verschwinden sie scheinbar ganz. Das Verhältnis des Kernes zum Faserwerk jeder einzelnen Zelle ist auch hier das oben geschilderte, nur entspricht die Längsachse des Kernes der Längsachse der Zelle und beide sind senkrecht zur Epithelbasis gestellt.

Ein merkwïrdiges Verhalten, auf welches Ehrmann schon wiederholt aufmerksam gemacht hat, zeigen gewisse Zellen oder besser gesagt Zellgruppen des Stratum spinosum und zwar gerade diejenigen, die an Größe alle anderen übertreffend, die zentralsten Anteile der Epithelleisten einnehmen. (Fig. 10, 13, 14.) Sie stoßen nach oben an das verbreiterte Stratum granulosum oder beim nässenden Condylom an die in regressiver Metamorphose befindlichen von Leukocyten durchsetzten usurierten, obersten Epithelschichten. Diese Zellen 
kommen nicht allein im breiten Kondylom vor, sondern sind überall dort vorhanden, wo Akanthose zugleich mit geänderten Verhältnissen der Verdunstung an der Oberfläche zu finden ist. Sie kommen außer im breiten Kondylom auch in spitzen Kondylomen, in Naevis, bei Variola, beim Epithelioma molluscum, Epithelialcarcinom der Haut und gewiß noch bei unzählig vielen anderen Prozessen vor.

Wir haben sie auch bei normaler menschlicher Haut, sowie in der Lidhaut des Rindsembryo gesehen. Diese Zellen färben sich mit allen Methylenblauarten, mit Fuchsin, nach der Weigertschen Methode annähernd zur Hälfte, weshalb sie Ehrmann hemichromatische Epithelzellen nennt. In jenen Fällen, wo die Vertrocknung verhindert ist oder eine Verhornung nicht stattfindet, färbt sich gewöhnlich die untere Hälfte stärker - katachromatische Zellen. Dort wo eine Vertrocknung oder Verhornung stattfindet, ist die obere Hälfte gefürbt - anachromatische Zellen. In der Haarscheide ist gewöhnlich Anachromasie, ebenso in der krustösen, syphilitischen Papel. Wo die Vertrocknung an einer kleinen Stelle stattfindet, in deren Umgebung jedoch eine Akanthose, wie z. B. bei Variola, können die gefärbten Zellpartien eine schräge Richtung gegen die Kruste eirmehmen, die gegen die Kruste gerichtet ist.

Hier wollen wir uns darauf beschränken, diese Zellen beim breiten Kondylom näher zu würdigen.

Dieselben sind in der Regel katachromatisch, d. b. es ist der der nächsten Fpithelbasis zugowandte Teil der Zelle gefärbt, während der abgewandte, also gewöhnlich der obere Teil wenig oder nicht gefärbt ist. An Präparaten, die nach Kromayer auf Epithelfasern gefärbt wurden, sieht man je nach Intensität der Entfärbung ctwas von cinander abweichende Bilder. Im äufersten Grade Aer Behandlung sieht man die oberen Anteile der Zellen scheinbar zu einem eine Wölbung darstellenden Flechtwerke von Fasern umgewandelt, das die perinukleare Höhle umscliließt und nach abwärts in die hyperchromatisch unteren Partien der Zelleiber übergeht. Diese sind entweder nach unten ausgebaucht oder laufen in Form eines Keiles aus, sind diffus oder feinkörnig intensiv gefärbt. Fpithelfasern ziehen stellenweise durch sie als stärker gefärbte Linien, die in die interspinalen liäume gelangen, als spinale Fortsätze noch immer ihre intensivere Färbung beibehalten, dabei plumper aussehen, als die mehr seitwäts gehenden. Der untere hyperchromatische Teil der Zelle ist nach oben konkav ausgehöhlt und umschließt mit 
dem oben erwähnten Gewölbe eine kugelförmige oder ovale Höhle, in welcher die Kerne verbildet und immer an die untere Wand der Höhle gegen den stärker gefärbten Zellpol gerückt erscheinen. (Fig. 10.) Bei solchen nässenden breiten Kondylomen, die an der Oberfläche eine starke Auflagerung von Leukocyten und einen reich lichen Zerfall von Epithelzellen der oberen Schichten zeigen, sieht man die rom starkgefärbten Teil des unteren Zellpoles ausgehenden interspinalen Fortsätze der hemichromatischen Zellen stellenweise verdickt, verkürzt, stalaktitenförmig abgerundet oder mit dem stärker gefärbten Pole zu einer Schichte von kugeligen oder maulbeerförmigen Körperchen zerfallen, welche die interspinalen Räume scheinbar ausfüllen, so daß unter Umständen verzweigte Figuren entstehen, die Ehrmann schon seinerzeit beschrieben hat urd von denen $\mathrm{J}$ arisch geglaubt hat, sie ständen mit den Melanoblasten in genetischem Zusammenhang, was gewiß nicht der Fall ist, weil sie gerade an den zentralsten Stellen des Kondyloms vorkommen, wo die Melanoblasten bereits gänzlich zu Grunde gegangen sind und nicht an jenen, wo sie noch erhalten sind. Sie kommen in den zentralsten Partien der breiten Kondylome und zwar nur jener Kondylome vor, die oberflächlich bereits zerfallen sind, während die Melanoblasten gerade in jenen Kondylomen am schönsten zu sehen sind, wo der Zerfall noch nicht soweit vorgeschritten ist. Ferner kommen die vergrößerten Melanoblasten ausschließlich oder nahezu ausschließlich in größerer Zahl ganz gewiß nur bei brünett gefärbtem Genitale vor, bei hellfärbigem gar nicht oder nur sehr spärlich, die oben beschriebenen Figuren aber ohne Unterschied auch bei ganz hellgefärbtem Genitale.

Die Leukocyten, welche in der Basalschichte vereinzelt in Form von rosenkranzförmigen Kernreihen zu sehen sind, sammeln sich im Stratum Malpighi und zwar gerade in der Schicht der hemichromatischen Zellen zu Haufen und Häufchen, welche die Epithelzellen verdrängen und sich kleine Höhlen bilden, in welchen sie mehr oder weniger zusammengeballt liegen, so daß das Kernconvolut ein maulbeerförmiges Aussehen darbietet. Stellenweise verdrängen sie den Zelleib der Epithelzellen und legen sich in die so gebildeten Nischen hinein, ähnlich den Osteoklasten. Gewöhnlich verdrängen sie den oberen ungefärbten Teil der hemichromatischen Zellen, 
so daß auch dies dafür spricht, daß dieser Teil der weichere, nachgiebigere ist. Außerdem liegen noch vereinzelte Leukocyten zwischen den hemichromatischen Zellen, da die ersteren bei der K r o mayerschen Färbemethode ebenfalls ein bißchen schrumpfen, so kann man ihre Lage zwischen den Zellen und in den Nischen derselben und ihre Beziehungen in den interspinalen Räumen ganz gut sehen.

Bekanntlich werden die pigmentierten Zellen in der Epidermis von manchen Autoren einfach als Wanderzellen (Cellules migatrices) bezeichnet und mit Leukocyten identifiziert. Das breite Kondylom ist wohl der klassische Boden, wo man sich von der Unrichtigkeit dieser Anschauung überzeugen kann. Die Melanoblasten sind Gebilde sui generis, die nichts mit Leukocyten zu thun haben, sondern als solche schon frühzeitig im Embryo an der Epidermisgrenze angelegt in die Epidermis einwachsen, während Leukocyten und Ehrlichsche Mastzellen in die Epidermis einwandern. Abgesehen von allen anderen Dingen ist schon die ganz enorme Größendifferenz zwischen Lcukocyten und Melanoblasten, die oft dicht nebeneinander liegen, ein schlagender Bewcis gegen diese Möglichkeit. Man vergleiche blob die Leukocyten auf Fig. 13 mit Melanoblasten auf Fig. 1, 2, 5, 6. Beide liegen in den interspinalen läumen und verdrängen bald die spinalen Fort. sïtze, bald ziehen sie zwischen ihnen durch.

Was nun die Struktur der hemichromatischen Zellen betrifft so ist im oberen Teile derselben anscheinend nichts zwischen den Epithelfasern, während nack unten zu eine sich stark färbende Substanz zwischen denselben absolut nicht zu verkennen ist. Wenn jedoch die Entfärbung nicht zuweit gedichen ist, so ist auch zwischen den Fasern der oberen Hälfte eine blad.3 gefärbte Zwischensubstanz nachweisbar, wie ja überhaupt in allen akanthotischen Epithelzellen, zwischon den Epithelfasern eine blaß gefärbte $Z$ wischensubstanz nicht zu verkennen ist, falls die Entfärbung nicht zu weit getrieben wurde. Werden die Präparate, ehe sie der Kromayerschen Fürbung unterzogen werden, mit Saffranin oder Alaunkarmin vorgefürbt, so fürbt sich die Grundsubstanz sehr gut und man kann deutlich die blauen Protoplasmafasern durch die rötliche Grundsubstanz ziehen sehen. 
Treibt man die Entfärbung der Epithelfasern zu weit, so erscheinen auch der hyperchromatische Pol und die Epithelfasern in der gleichmässigen Grundfarbe; allerdings sind die hemichromatischen Zellpolen auch da stärker gefärbt. Eine Färbung des perinukleären Raumes ist uns nie gelungen.

Ehrmann hat schon früher die Erscheinung der Hemichromasie darauf zurïckgeführt, daß in dem wenig sich färbenden Teile der Zelle eine größere Flüssigkeitsmenge enthalten ist, als im stärker sich färbenden und sieht dies für einen Beweis dafür an, daß beim nässenden Kondylom, wo an der Oberfläche bekanntlich eine intensive Flüssigkeitsausscheidung stattfindet, die oberen Zellpole einen Überschuß an Wasser aufnehmen, um denselben dann auszuscheiden; deshalb färben sich dieselben schwächer, deshalb sind die Schrumpfungserscheinungen bei der Darstellung der Epithelfasern stärker und deshalb ist die Zwischensubstanz schwieriger nachzuweisen.

Die Hemichromasie der Epithelzellen tritt bei allen Färbungen mit basischen Anilinfarbstoffen zu Tage; sie ist etwas von der Methode Unabhängiges. Dagegen sind die anderen Erscheinungen wesentlich von der Methode abhängig. Die Bildung des perinukleären Raumes ist zweifellos ein Kunstprodukt, erzeugt durch die Kromayersche Modifikation der W eigertschen Fibrinfärbungsmethode; denn sie fehlt an Präparatstellen, wo die Entfärbung nicht bis zur vollsten Faserfärbung gediehen ist und kommt einesteils durch Schrumpfung und Verkrümmung des Kernes, audrerseits durch Retraktion des Zellprotoplasmas und seiner Fäden vom Kerne zu stande.

Als Beweis kann folgendes dienen:

An jenen Stellen, wo die Entfärbung des oberen chromatischen Teiles der Zellen gerade nur soweit gediehen ist, daß man eben die Protoplasmafasern sieht, dort ist der Kern bläschenförmig, kugelig oder oral und von einem kaum merkbaren Spalt rings umgeben. Der zwischen Kern und interspinalem Raum befindliche Anteil des Protoplasmas ist noch ziemlich breit. An jenen Stellen dagegen, wo die Fasern scharf zu sehen sind, ist zwar der obere achromatische Anteil des Zellprotoplasmas als dünne scheinbar nur aus einem Geflecht ron Protoplasma- 
fasern bestehende Schale zu erkennen, während der untere Anteil noch ziemlich mächtig erscheint, wenn auch kieiner als an solchen Präparaten, die nach anderen nicht schrumpfenmachenden Methoden gefärbt sind.

Ehrmann hat an anderen Stellen gezeigt, daß die Fasern umso schärfer hervortreten, je wasserhaltiger das Protoplasma ist und je mehr dasselbe Gelegenbeit hat bei Auilinxylolbehandlung zu schrumpfen. Dies bestätigt sich hier aufs neue, ebenso wie es deutlich wird, dal die Epithelzelle nicht ein bloßes Flechtwerk ron Fasern ist, sondern daß zwischen den Fasern sich noch eine Substanz befindet, die je nacb ihrem Wassergehalte bei der Präparation mehr oder weniger schrumpit und an die Faser herangezogen wird. Vielleicht ist die Struktur am besten beschrieben, wenn man sagt: Die Epithelzelle bestehtaus einer Substanz, diean verschiedenen Stellen des Zellprotoplasmas verschieden stark wasserhältig ist, in welcher sich faserförmige Verdichtungen finden, welchedieinterzellulären Räume von Zelle zu Zelle überbrückend als Spiualfortsätze in die Frscheinung treten.

Der Wasserreichtun der Epithelfasern scheint weniger zu variieren als der der übrigen Zellsubstanz.

Die interfibrilläre Grundsubstanz der Epithelzellen kanu man am schönsten an jenen Zellen sehen, welche sich durch Degencration zu Perlkugeln umwandeln, wie es auf Fig. 12 sichtbar ist. An der kugelig werdenden 7elle kann man eine ziemlich gut sich fürbende Hohlkugel von degenerierter \%wischensubstanz unterscheiden, in welcher der Kern liegt und innerhalb der kugelschalenförmig geformten Zwischensubstanz sieht man sehr deutlich den zum Teile radiären Verlauf der spinalen Fortsätze, welche zu den platgedrückten, die Kugel zwiebelschalenförmig ungebenden Zellen führen. Auch an diesen Tellen kann man noch eine Spur von Hemichromasie sehen und zwar in derselben Riching, nach welcher der hemichromatische Pol einer Nachbarzelle gerichtet ist. Die uibrigen abgeplatteten Zellen stellen alle scheinbar nur ein Geriustwerk von Fasern dar, welche Kerne umschließen. Hier kann man mit Sicherheit sagen: Die Degeneration der Zellsubstanz, welche 
zur Bildung der Perlkugeln führt, geht ron der Zwischensubstanz aus, beruht also nicht auf der Umwandlung der Protoplasmafasern, gewiß nicht allein.

Bemerkenswert ist die Umwandlung der hemichromatischen Zellen in den oberflächlichsten Schichten der Oberhaut, unmittelbar unter der erodierten Papeloberfläche, die an Stelle der zu Grunde gegangenen Körnerschicht sich finden. Die Kerne dieser Zellen werden klein, spärlich, wenig färbbar und unansehnlich. Die Faserung des Protoplasmas ist nur stellenweise und in Spuren vorhanden; dafür stellt der obere schwach gefärbte Teil der Zelle ein schwammiges Gerüstwerk von homogenen, wenig sich färbenden Scheidewänden und Balken dar, zwischen welchen scheinbar große und leere Lücken vorhanden sind, in denen hie und da noch ein blasser Kernrest zu sehen ist (Fig. 11).

Der Zellumriß ist undeutlich und wird noch am besten durch die erhaltenen stark chromatischen stalaktitenähnlichen Reste der unteren Zellenhälfte angedeutet. Diese ganze Erscheinung läßt sich wohl nur durch weitere Zunahme der Flüssigkeitsmenge im Zellprotoplasma, welche sich in Vacuolen ansammelt und das übrige Protoplasma zu einem Netzwerk verdrängt, erklären. Es ist der äußerste Grad der hydropischen Degeneration bei noch erkennbarer Zelle.

\section{Melanoblasten und Epithelfasern.}

Im Jahre 1886 hat Ehrmann bereits verzweigte, große Zellen beschrieben, welche zwischen den wenig oder ganz unpigmentierten Epidermiszellen des breiten Kondyloms liegen und eine große Ähnlichkeit mit den Pigmentzellen bei Amphibien zeigen. Die Genese dieser Zellen wollen wir hier außer acht lassen; es wurde darüber bereits anderwärts von $\mathrm{Ehr}$ mann ausführlich gesprochen. Hier wollen wir nur den Einwänden begegnen, welche von jenen Autoren vorgebracht wurden, die sie nicht für Zellen gelten lassen wollen, sondern sie für A usgüsse der interzellulären Räume erklären.

So einfach und schlagend diese Anschauung widerlegt wurde, so begegnet man ihr doch wieder, ein Beweis dafür, 
wie zersplittert die wissenschaftliche Arbeit in der neuesten Zeit ist, da Dinge, die zehnmal widerlegt worden sind, immer wieder aufs Neue behauptet werden, nicht weil man neue Gründe dafür beigebracht hätte, sondern weil man die vorgebrachten Gegengründe nicht kennt.

Die interzellulären Räume sind nicht, wie schon anderwärts dargelegt wurde, $\mathrm{R} \ddot{\mathrm{o}} \mathrm{h} \mathrm{r}$ en, sondern poly g on ale $\mathrm{H}$ oh lplatten. Ausgüsse derselben könnten also nicht fadenförmig, sondern nur plattenförmig sein, und wenn sie auch auf dem Querschnitt bei oberflächlicher Betrachtung als Fäden imponieren könnten, so müßten doch genug Bilder vorkommen, welche die Platten in größerer oder geringerer Ausdehnung in der Schnittebene oder parallel zu derselben zeigen. Ferner müßte bei der Verschiebung der Mikrometerschraube bei irgendwie dickeren Schnitten der als Faden imponierende optische Querschnitt sich im Sehfelde hin und her verschieben und zum Schlusse wäre es nicht möglich, daß über einer dem Beschauer zugewendeten Fläche einer Epidermiszelle ein bedeutend scharf kontourierter pigmentierter Faden sichtbar wäre, sondern es mübte eine pigmentierte Fläche zu sehen sein. Die eine Epithelzelle scheinbar umschließenden Pigmentsäume dürften auf dickeren Schnitten, wenn sie Plattendurchschnitte wären, bei Verstellung der Mikrometerschraube nicht plötzlich verschwinden, sondern müßten solange sichtbar bleiben, bis die Platte in allen optischen Querschnitten eingestellt war. Dies sind die negativen Argumente, die positiven werden folgen.

Bekanntlich besteht aber noch eine zweite Peihe von Anschauungen, welche zwar die Melanoblasten für Zellfiguren hält, ihre Selbständigkeit aber leugnet, von denen eine von K r o mayer entwickelt wurde. Nach ihm sind die Chromatophoren nichts anderes, wie epitheliale, dem Faserverlauf der Epithelien entsprechende Figuren, als der Ausdruck der beginnenden Pigmentation der Epithelzellen. Die kleineren sternförmigen Melanoblasten sind $\mathrm{Kr}$ o m a y e r nichts anderes als sternförmige Epithelzellen, deren Fasern sich zu Pigment umgewandelt haben, die größeren, deren $\ddot{\Lambda}$ ste mehrere Epithelzellenbreiten lang sind, wären nur Pigmentfiguren, die durch Pigmentation 
von in derselben Richtung verlaufenden Fasern mehrerer Epithelzellen zu stande kommen.

Wie haltlos diese Anschauung ist, zeigen die Abbildungen (Fig. 1, 2, 3, 5, 6. 7, 9), die aus Präparaten gewonnen wurden, wo die Epithelzellen überhaupt kein Pigment enthielten, also eine Vortäuschung von Pigmentzellen durch Epithelzellen totaliter ausgeschlossen ist. Schon bei halbwegs aufmerksamer Besichtigung der Präparate erkennt man die Selbständigheit der Pigmentzellen und ihre volle Unabhängigkeit von den Epithelzellen. Man sieht wie die Richtung ihrer Fortsätze mit der Richtung der Epithelfasern nichts zu tun haben. Die Epithelfasern verlaufen quer bald über, bald unter dem Melanoblastenfortsatz, sie treten $n$ irgends in Verbindung mit dem Zellkörper oder Ausläufern der Melanoblasten, deren Protoplasma vollständig frei ron jeglicher Faserstruktur ist. Auch das Pigment in den Melanoblasten, das bald grob bald feinkörnig ist, zeigt hier keine Anordnung zu Reihen, weil es ja nicht mehr in Strömung nach den Epithelzellen hin begriffen ist, mit denen die Melanoblasten sonst zusammenhängen. Es ist zum Teil in ganz unregelmäßiger Anordnung sichtbar (Fig. 1, 2, 3), zum Teil zeigt sich eine gewisse Gruppierung um den Kern und an der Oberfläche der Pigmentzellen (Fig. 5). Manchmal ist die Anordnung des Pigmentes in den Melanoblasten eine derartige, daß sich das nicht pigmentierte Protoplasma in Form kleiner pigmentloser Fleckchen von dem wabig oder schaumig angeordneten Pigmentkörnchen abhebt. (Fig. 1.)

Die Kerne sind deutlich blau gefärbt und unterscheiden sich oft durch die Intensität dieser Färbung von den weniger intensiv blau gefärbten Kernen der Epithelzellen.

Die Zugehörigkeit eines Melanoblasten-Kernes zum betreffenden Melanoblasten gibt sich auch dadurch kund, daß der Kern allseits rom pigmentierten Zelleib der Melanoblasten umschlossen ist und der in einem Interzellularraume samt seinen Ausläufern liegt, und daß beides, pigmentierter Zelleib und Ausläufer in einem gemeinsamen Hof, der durch Zurückweichen der Epithelzellen gebildet wird, frei liegen, also eine Verwechslung mit Kernen von Epithelzellen vollkommen ausschließen. Man 
bekommt dadurch Bilder, in denen es den Auschein hat, als ob der Melanoblast die Epithelfasern auseinanderdränge, um sich Platz zu machen. (Fig. 7.)

Die Fortsätze der Melanoblasten haben alle möglichen Gestalten. Sie verlaufen bald geradlinig, bald gekrümmt, nicht selten zickzackförmig gebrochen, was ein weiterer Beweis dafür ist, daß die Fortsätze nicht aus zufällig in einer Richtung verlaufenden Epithelfasern zusammengesetzt sind.

Viele Pigmentfortsätze endigen mit Knöpfen entsprechend der sistierten Pigmentströmung von den Melanoblasten zu den Epithelzellen hin.

Ganz und gar unmöglich ist es aber die Krom a yersche Theorie auf jene Melanoblasten anzuwenden, die an der Grenze zwischen Cutis und Epidermis liegen, oft von der Epidermis durch einen freien Raum abgehoben (Fig. 6). Diese können unmöglich Epithelfaserfiguren darstellen, da sie absolut keine Spur eines Zusammenhanges mit Epithelfasern zeigen. Die Fortsätze dieser Melanoblasten verlaufen nicht selten in einer ziemlichen Entfernung von der Epithelgrenze. Auf diese Tatsache hat außer Ehrmann auch Herxheimer zumal beim Pemphigus vegetans hingewiesen, der ja ähnliche Verhältnisse setzt, wie das breite Kondylom. Diese parallel zux Epithelgrenze verlaufenden Melanoblasten können auch schon deshalb nicht zu Pigment zerfallenen Epithelprotoplasmafaser entsprechen, weil in der Basalschichte die Protoplasmafasern nur senkrecht zur Epithelgrenze verlaufen. Nur die kurzen interspinalen Fasern verlaufen parallel za dicser Grenzo; diese können aber unmöglich pigmentierte Gebilde, die mehrere Epithelzellen breit sind, samt ihren Ausläufern vortäuschen, auch liegen sie im Epithel und nicht unterhailb derselben.

Die im Bindegewebe der Papillen verlaufenden Fortsätze zeigen nur den Unterschied von den im Epithel verlaufenden, der ihnen dadurch aufgeprägt wird, daß die letzteren ihre Fortsätze in den Interspinalräumen, die ersteren in den Interstitien des Bindegewebes zeigen.

Sonst sind Zelleib und Pigment von derselben Beschaffenheit. Im Bindegcwebe sollten sic als solche bestehen, im Epi- 
thel Trugbilder sein, die durch Pigmentation von Epithelfasern zustande kommen?

Die mit Methylenblau-Ferricyankalium gefärbten Präparate zeigen dieselben Verhältnisse, nur viel weicher, weniger in starre Formen gegossen, wegen der viel weniger eingreifenden und schrumpfen machenden Methode Un nas. Dafür sind dementsprechend auch die erhaltenen Bilder der Protoplasmafaserung weniger prägnant. (Fig. 9.)

Aber überall machen die Melanoblasten den Eindruck des Selbständigen, von den Epithelzellen vollkommen Unabhängigen. Nirgends zeigt sich in den Melanoblasten eine Spur von Epithelfaserung. Aber nicht deshalb, weil sich, wie Krom a y er meint, sämtliche Fasern in ihnen zu Pigment umgewandelt haben, sondern weil die Melanoblasten a priori keine Epithelfasern besitzen, da sie ja Abkömmlinge des M e s oderms sind. Es ist dies somit ein weiterer direkter Beweis für die Abstammung der Pigmentzellen aus dem Mesoderm, die Ehrmann schrittweise bei dem sich entwickelnden Embryo nachgewiesen hat.

Bindegewebszellen, sowie andere aus dem Mesoderm abstammende Zellen zeigen niemals Epithelfaserung.

Zum Schlusse noch folgende Erwägung: Wenn die Pigmentfortsätze wirklich eine zusammenliegende Suite von in Pigment umgewandelten Epithelfasern wären, so hätten wir es nicht mit dünnen, fadenförmigen, auf dem Durchschnitt spulrunden Gebilden zu tun, sondern es wären unregelmäßige Flächen oder flügelförmige membranähnliche Gebilde, was die Fortsätze in Wirklichkeit nicht sind.

Wenn man die fadenförmigen Fortsätze zum Beispiel über die Seitenwand einer Zelle verlaufen sieht, so daß der Kern bei einer bestimmten Einstellung, der über ihm verlaufende Fortsatz bei einer anderen Einstellung scharf sichtbar ist, so kann man sich überzeugen, daß der Fortsatz als eigenes in sich geschlossenes fadenförmiges Gebilde ganz anders verläuft als die Epithelfasern.

Dies sind die positiven Argumente für die Zellennatur der Chromatophoren. Wer danach noch von bioßen Pigmentoder Chromatophorenfiguren sprechen kann, sieht wohl die Zellen vor lauter Fasern nicht. 
Jetzt noch einige Worte zur Ansicht Kromayers, daß das Pigment aus zerfallenen Epithelfasern entsteht.

In der äußersten Peripherie des breiten Kondyloms, am Übergange zur normalen Haut, sind auch die Epithelzellen pigmentiert und es habeu namentlich die Basalzellen das Pigment an der der Hautoberfläche zugewendeten Seite in Kappenform, wie bereits vielfach beschrieben wurde. Von diesen pigmentierten Kuppen der Basalzellen gehen die Spinalfasern genau so zu den benachbarten Zellen, wie an nicht pigmentierten Stellen. Sie lassen sich als blaue Linien in das gelbgrüne Innere der pigmentierten Kuppen verfolgen (Fig. 3, 4), ohne daß innere Pigmentkörnchen in ihrer Richtung angeordnet wären.

Das letztere kommt zwar vor, findet aber in der Annahme Eh r ma n n s eine genügende Erklärung, daß die Pigmentströmung von der Basis zur Oberfläche längs den Eipithelfasern erfolgt. Genau so wie das Pigment in den Furchungskugeln und embryonalen Zellen der originär pigmentierten Frosch- und Axolotlembryonen bei jeder Phase der Fntwicklung seine Lage ändert und dabei in linearer Richtung strömt. (Siehe die in der Bibliotheka medica erschienene Arbeit $\mathrm{E}$ h r m an $\mathrm{s}$ : Das melanotische Pigment und die pigmentbildenden Zellen etc.)

$\mathrm{D}_{\mathrm{a}} \mathrm{B}$ bei pigmentierten Epithelzellen die Faserung undeutlich ist, ja manchmal ganz unsichtbar, erklärt sich ungezwungen aus der mangelhaften Schrumpfung der mit Pigment erfuilten Fpithelzellen, deren Protoplasma an den Pigmentkörnern gewissermaßon ein Stützwerk besitzt, das dasselbe am Schrumpfen hindert. Ganz dasselbe ist ja in der Keratohyalin schicht der Fall, von der man behauptet hat, daß überhaupt keine Fasern in ihr vorkommen; und doch sind dieselben bereits nachgewiesen worden, wie auch unsere Abbildungen beweisen. Die Fasern sind eben hier schwerer darstellbar, weil die Zellen der Körnerschichte durch die Keratohyalinkörner am Schrumpfen stark gehindert sind. Einmal sind es die Keratohyalinkörner, ein andermal die Pigmentkörner, die bewirken, daß die Darstellung ảer Protoplasmafasern schwieriger gelingt. Bei entsprechender Awwendung der Kromayerschen Methode kann man beide Male die Fasern deutlich sichtbar machen, was bei den Mclano blasten niemals gelingt. 
Dazu kommt noch, daß die Pigmentkörnchen keineswegs so einfache Gebilde sind, wie sie Kromayer abbildet, als schwarze Punkte oder Striche, sondern sie bestehen zweifellos, wie Ehrmann wiederholt nachgewiesen und Unna auch bestätigt hat, aus einer ungefärbten Grundsubstanz, in welcher das bouteillengrüne Pigment aufgelöst ist.

Der große Unterschied zwischen Melanoblasten und pigmentierten Epithelzellen liegt darin, daß erstere, wenn sie auch zuweilen, wie oben gesagt wurde, die Pigmentkörnchen in Reihen angeordnet zeigen können, niemals Epithelfasern besitzen, was auch K r o ma y e r nicht nacbweisen konnte, während letztere deutlich Protoplasmafaserung zeigen.

Die reihenförmige Anordnung der Pigmentkörnchen in den Chromatophoren der Cutis der Fische, also in Gebilden, die gewil nicht epithelialen Ursprungs sein können, ist schon seit langem durch die Untersuchungen von Solger, Zimmermann, Eberth und Ballowitz bekannt. Ebenso ist es bekannt, daß die Pigmentreihen in denselben die Wege darstellen, auf welchen die Pigmentkörnchen (Tröpfchen) sich bewegen.

\section{Schlußsätze :}

1. In der Epidermis des breiten Kondylomerfolgt neben einerVergrößerung der Epithelzellen (Akanthose) auch eine Vergrößerung der Melanoblasten.

2. Dic Veränderungen, welchedieEpithelzellen hiebei erleiden, machen dieselben mit der Zeit unfähig, Pigment von den Melanoblasten aufzunehmen.

3. Während in der äußeren Peripherie des hreiten Condyloms das Pigment in den Epithel. zellen in gleicher Weise wie die Melanoblasten vermehrt ist, fehlt imZentrum des breiten Condyloms das Epithelpigment, die Melanoblasten, und sind zugrunde gegangen.

4. Die Melanoblasten der Epidermis sind eigene Zellen mesodermalen Ursprungs und weder Ausgüsse der interzellularen Räume noch optische Trugbilder der Epithelzellen. 
5. Die beim breiten Kondylom, wie auch bei and erenProzessen vorhandene Hemichromasie beruht auf einer verschiedenen Verteilung des Wassers im Zelleib.

Ihr Zelleib wie der der übrigen Epithelzellen besteht aus Protoplasmafasern und verschieden wasserreicher Grundsubstanz.

6. Die Lage der hemichrumatischen Pole hängt vom Verdunstungs-resp. Verhornungsprozess der 0 berfläche ab.

7. Aus Leukocytenkönnen keine Melanoblasten entstehen.

\section{Nachtrag.}

An dem Materiale, welches der eine von uns $(0$ ppenhei m) auf einer Reise in Indien gesammelt hat, konnten wir feststellen, da\} in der leprösen Haut der dunklen Rassen ganz ähnliche Bilder der Melanoblasten erhalten werden, wie bei Syphilis; wir beabsichtigen darüber in einem besonderen Artikel zu berichten.

\section{Literatur.}

B a 110 witz. Verhandlungen des anatomischen Kongresses zu Göttingen 1893.

Beneke. Epithelfaserung der menschlichen Oberhaut. Verhandl. d. Gesellschaft deutscher Naturforscher und Ärzte 1894. Bd. II.

Eberth. Die Nerven der Chromatophoren. Anatom. Kongreb Göttingen 1893.

Eddowes. Über die Natur der Herxheimerschen Spiralen der Oberhaut. Monatsh. f. prakt. Derm. 1890. Bd. II.

Ehrmann. a) Zur Physiologie u. Pathologie des Hautpigmentes. Archiv f. Dermatologie. 1885/86.

b) Über Entwicklnng und Wanderung des Pigmentes. Archiv f. Dirmatologie. 1891.

c) Über die Entwicklung des Pigmentes bei den Amphibien. Zentralblatt für Physiologie. 1895.

d) Zur Physiologie der Pigmentzelien nach Studien über Farbenwechsel der Amphibien. Arch. f. Derm. 1892.

e) Die Weigertsche Fibrinfärbungsmethode und das Studium des Oberhautpigmentes. Arch. f. mikroskop. Anatomie. Bd. XLIII. 
f) Das melanotische Pigment und die pigmentbildenden Zellen des Menschen und der Wirbeltiere. Bibliotheka medica. D. II. Heft 6. g) Über Hautentfärbungen durch sekundär syphilitische Exantheme. Arch. f. Derm. 1891.

h) Verhandlungen der deutschen dermatologischen Gesellschaft. 1892. pag. 307.

i) Zur Histologie der Syphilide. Wiener klin. Rundschau. 1886.

k) Bericht des intern. Kongresses f. Dermatologie in Wien 1893.

l) Einiges über die Rolle der Melanoblasten bei der Syphilis. Festschrift F. J. Pick 1898.

m) Zur Pathologie der Syphilide. Wr. kl. Rundschau. 1897. Nr. 25.

Ernst. Studien über die normale Verhornung mit Hilfe der Gramschen Methode. Arch. f. mikr. Anatom. Bd. XLVII. Heft 4.

Fisch I. Arch. f. mikroskop. Anatom. 1896.

Garten, S. Die Interzellularbrücken der Epithelien und ihre Funktion. Archiv. f. Physiologie. 1895/96.

Halpern. Über das Verhalten des Pigmentes in der Oberhaut des Menschen. Arch. f. Derm. 1891. Bd. XXIII.

Herxheimer. a) Über eigentümliche Fasern in der Epidermis. Arch. f. Derm. 1889. Bd. XXI.

b) Über die Struktur des Protoplasmas der menschlichen Epidermiszelle. Arch. f. mikr. Anatom. u. Entwicklungsgeschichte. 1899. Bd. LIII.

c) Nachtrag und Berichtigung. Ibidem. 1899. Bd. LIV.

d) Über Pemphigus vegetans nebst Bemerkungen über die Natur der Langenharsschen Zellen. Arch. f. Dermatologie. 1896. Bd. XXXVI. Herxheimer u. Müller. Über die Bedeutung der sogenannten Epidermisspiralen. Arch. f. Derm. u. Syph. XXXVI. 93.

Jarisch. a) Über die Anatomie und Entwicklung des Oberhautpigmentes beim Frosche. Arch. f. Derm. 1891.

b) Zur Anatomie und Herkunft des Oberhaut- und Haarpigmentes. Arch. f. Derm. u. Syph. 1891.

c) Über die Bildung des Pigmentes in den Oberhautzellen. Arch. f. Derm. 1892. Bd. XXIV.

Jadas ohn. Verhandlungen der deutschen dermatologischen Gesellschaft. 1892, pag. 307.

Kromayer. a) Zur patholog. Anatomie der Psoriasis. Arch. f. Derm. 1890. Bd. XXII.

b) Die Protoplasmafaserung der Epithelzellen. Arch. f. mikroskop. Anatomie. Bd. XXXIX.

c) Über die Deutung der von Herxheimer im Epithel beschriebenen

Fasern. Monatshefte f. prakt. Derm. 1890. Bd. XI.

d) Oberhautpigment der Säugetiere. Arch. f. mik. Anat. Bd.XIIII. 1893.

e) Die Parenchymhaut und ihre Erkrankungen. Archiv f. Entwicklungsmechanik. 1899. Bd. VIII

$f)$ Finige epitheliale Gebilde in never Auffassung. Beiträge zur Pigmentfrage. Dermatol. Zeitschrift. 1897. Bd. IV. 
Mertsching, S. Histologische Studien über Keratohyalin und Pigment. Virchows Archiv. Bd. CXVI. 1889.

Meyerson. Zur Pigmentfrage. Virchows Arch. Bd. CXVIII. 1889. N e umann, J. Histologie der Syphilide. Arch. f. Derm. 1885.

Rabl, H. Bleiben die Protoplasmafasern in der Körnerschicht der Oberhaut erhalten? Arch. f. Derm. 1897. Bd. XLI.

Derselbe. Pigment und Pigmentzellen in der Haut der Wirbeltiere. Ergebnisse der Anatomie und Entwicklungsgeschichte. 1896.

Riehl. Zur Kenntnis des Pigmentes im menschlichen Haar. Arch. Derm. 1884. Über Leucoderma syph. Med. Jahrb. 1884.

Schütz. Arch. f. Derm. Bd. XXXVI.

Solger. Zur Kenntnis der Pigmentzellen. Anat. Anzeiger. 1891.

Unna. a) Fortschritte der Hautanatomie. Das Pigment der Haut. Monatshefte f. prakt. Derm. Bd. VIII. Nr. 8. 1889.

b) Die Färbung der Epithelfasern. Monatshefte f. prakt. Derm. Bd. XIX. Nr. 2.

c) Die spezifische Färbung des Epithelprotoplasmas. Monatshefte f. prakt. Derm. 1894. Bd. XIX.

d) Über das Wesen der normalen und patholog. Verhornung. Monatsh. f. prakt. Derm. 1897.

Zi mmermann. Über die T'eilung der Pigmentzellen. Arch. f. mikroskop. Anat. 1890. Studien über Pigmentzellen. Ibidem.

\section{Erklärung der Abbildungen auf Taf. IX-XI.}

Fig. 1. Melanoblasten aus dem unteren Ende eines Kpithelzapfens im breiten Kondylom eines brünetten weiblichen Individuums. Fixierung in absolutem Alkohol, Härtung in Alkohol, Paraffineinbettung. Schnittdicke $4 \mu$. Färbung auf Epithelfasern nach Kiromayer. a Melanoblastenfortsätze die Epithelfasern kreuzend. Der Schnitt stammt aus der Zone $b$ (s. die Textabbildung).

Fig. 2. Aus demselben Präparate. a Melanoblastenfortsatz in einem freien Interzellularraum liegend. $b$ Ein im rechten Winkel abbiegender Melanoblastenfortsatz, dabei die Protoplasmafasern einer Epithelzelle kreuzend, bezichungsweise quer über dieselben hinlaufend.

Fig. 3. Schnitt aus der Zone a. Dicke $5 \%$. Färbung wie bei Fig. 1. Melanoblasten an der Cutisgrenze, zum Teil mit ihren Fortsätzen in das Epithel hineinragend. Pigmentkuppen der Basalzellen. Epithelfaserung in der Keratohyalinschicht. Von den pigmentierten Kuppen gehen blaue Epithelfasern aus.

Fig. 4. Aus derselben Zone. Dicke $4 \%$. Durch die Pigmentkuppen ziehen Epithelfasern. a Melanoblast mit einem Fortsatze im Bindegewebe mit einem zweiten im Epithel. Man vergleiche denselben mit der Epithelzelle b, welche eine gewisse Ähnlichkeit mit $a$ hat, aber trotz ihrer Pigmentierung deutlich Protoplasmafasern zeigt, namentlich in der Randzone des Protoplasmas. 
F i g. 5. Tangentialschnitt durch den unteren Teil eines Epithelzapfens. Färbung wie oben. Schnittdicke $5 \mu$. Melanoblasten dicht gedrängt. $a$ Melanoblastenfortsatz winkelig nach oben umbiegend. $b$ Spindelförmiger Melanoblast an der Grenze zwischen Cutis und Epithel. Zone $b$ in einer Lücke liegend, die durch Retraktion des Bindegewebes der Papille entstanden ist. Auch hier sieht man an mehreren Stellen Kreuzung der Melanoblastenfortsätze mit der Epithelfaserrichtung.

F ig. 6. Melanoblast unter der Epithelgrenze liegend, seine Fortsätze nach oben ins Epithel sendend. Schnittdicke $3 \mu$.

Fig. 7. Melanoblast mit seinem Körper die Epithelfasern auseinanderdrängend. Schnittdicke $3 \mu$.

Fig. 8. Wurde aus technischen Gründen weggelassen.

Fig. 9. Epithelfaserfärbung nach Unna (Polychromes Methylenblau, $1 \%$ Ferricyankaliumlösung). a Melanoblast an der Epithelgrenze; die Epithelfasern dessen Fortsatz kreuzend. Schnittdicke $5 \mu$.

Fig. 10. Schnitt aus der nässenden Papel. Fixiert in Alkohol. Fảrbung nach Kromayer. Dicke $5 \mu$. Hemichromatische Zellen; $a$ Leukocyten in Ausbuchtungen der Eipithelzellen liegend. $b$ Perinukleäre Höhlen. Die Kerne an die untere Wand gedrängt.

F'ig. 11. Aus demselben Präparat. In der Nähe der nässenden Oberfläche. Zunahme der Zellenverflüssigung. $a$ Stalaktitenartige Fortsätze.

Fig. 12. Perlkugelbildung. Sämtliche Bilder wurden mit Ölimmersion Reichert erhalten.

Fig. 13. Eine ältere Zeichnung Ehrmanns. Färbung mit wässrigem Methylenblau. Mittlerer und oberer Teil eines Durchschnittes einer Reteleiste. Man sieht die polynukleären Leukocyten auf ibrer Wanderung begriffen. Dieselben sind in Höhlen angesammelt, die zum Teile noch in die Schichte der hemichromatischen Zellen fallen, zum Teil an der Oberfläche liegen. Man sieht, wie die Leukocyten das Protoplasma der hemichromatischen Zellen verdrängen und wie durch die Leukocytenansammlung die Hornschichte abgestoßen wird. Vergrößerung: Reichert Okul. 3, Obj. 8.

F i g. 14. Ältere Zeichnung Ehrmanns. Hemichromatische Zellen nach der Weigertschen Fibrinmethode gefärbt. (Dasselbe Resultat gibt auch die Färbung mit Anilin-Fuchsin). Aus dem Retezapfen einer exkoriierten und nässenden, von einer pyogenen Schichte bedeckten Stelle des breiten Kondyloms. Man sieht in den oberen Lagen Zerfall des hemichromatischen Poles zu kleinen maulbeerförmigen Gebilden. Die unteren Zellen zeigen noch die stalaktitenförmigen nicht zerfallenen Fortsätze wie Fig. 10, 11, 13. 1/12 Ölimmersion. immersion.

Fig. 15. Polynukleäre Leukocyten in Zellaushöhlungen. 1/12 Öl- 

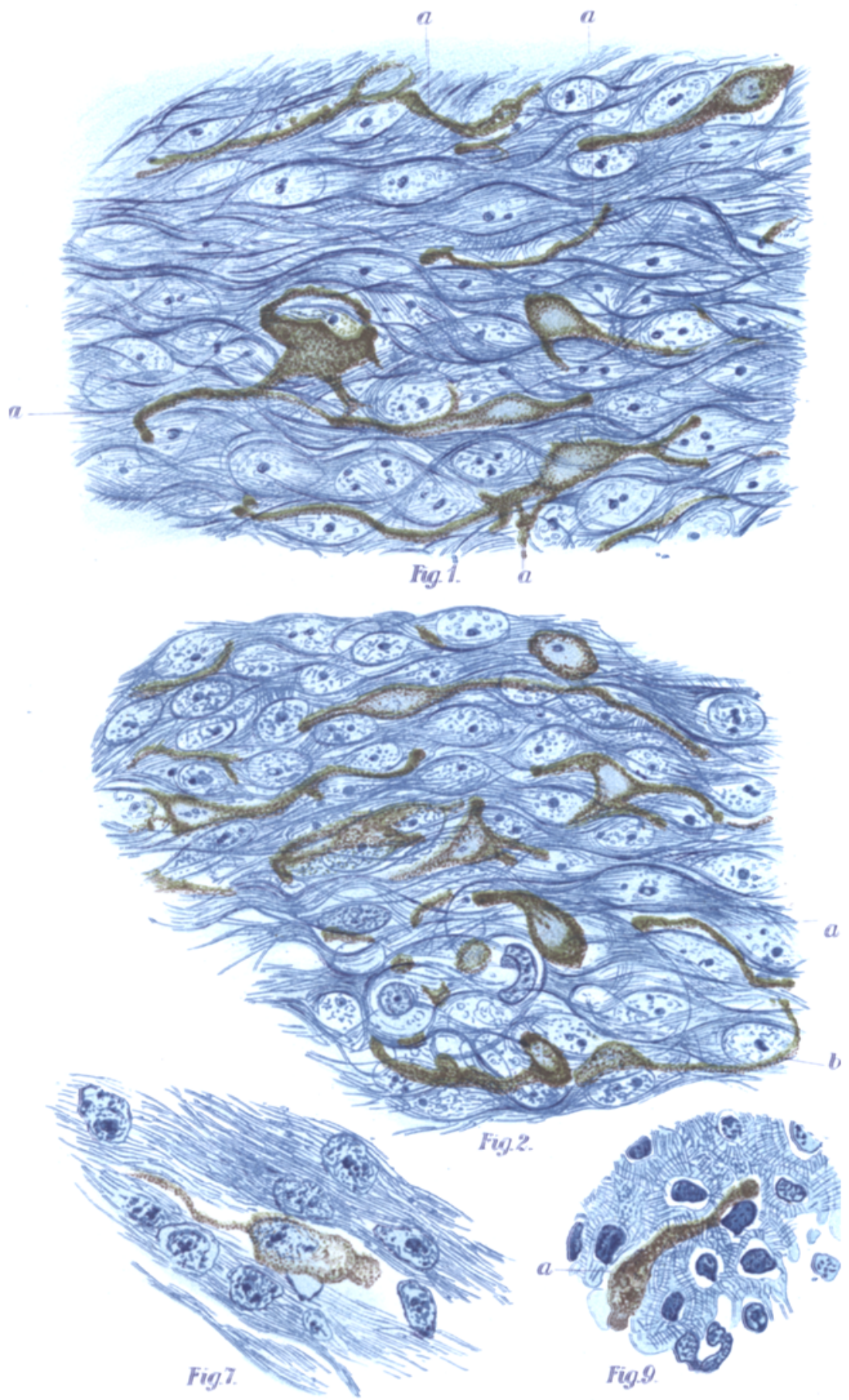

Ehrmann- Oppenheim : Ũ̄ber Melanoblasten. 


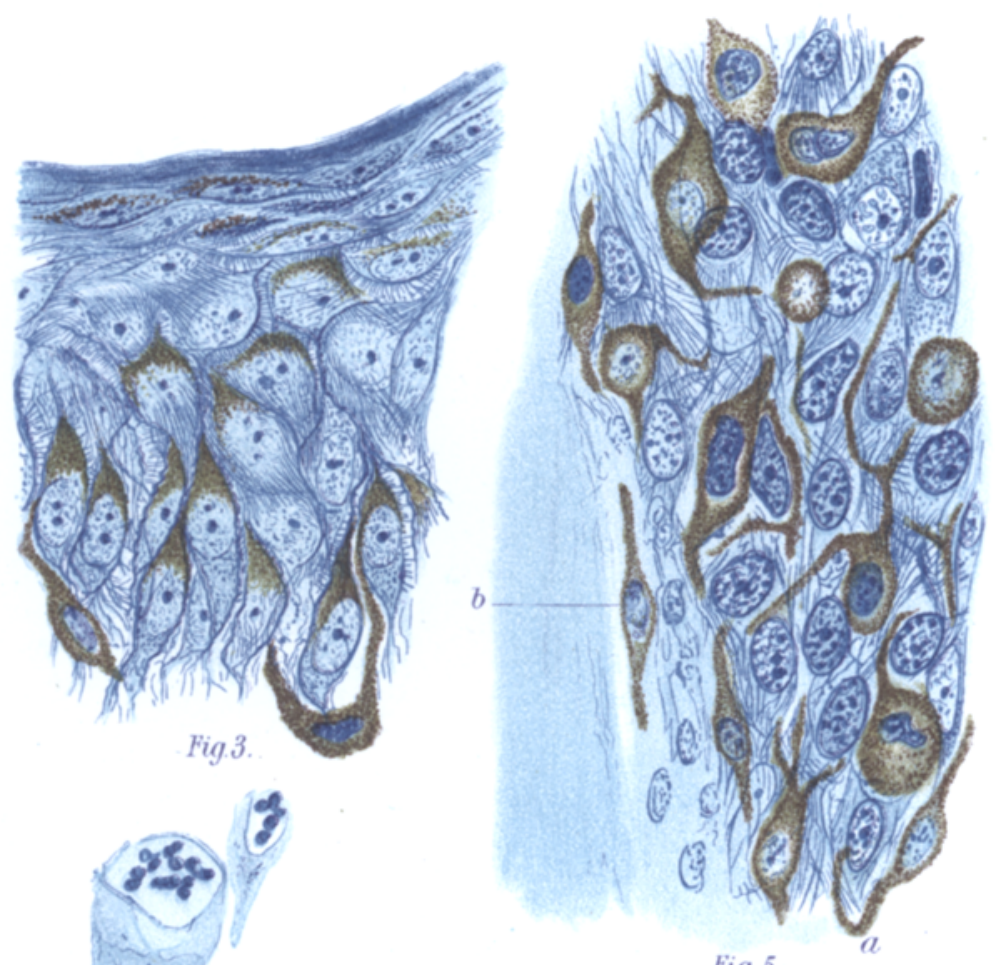

Fig. 5.

Fig.15.
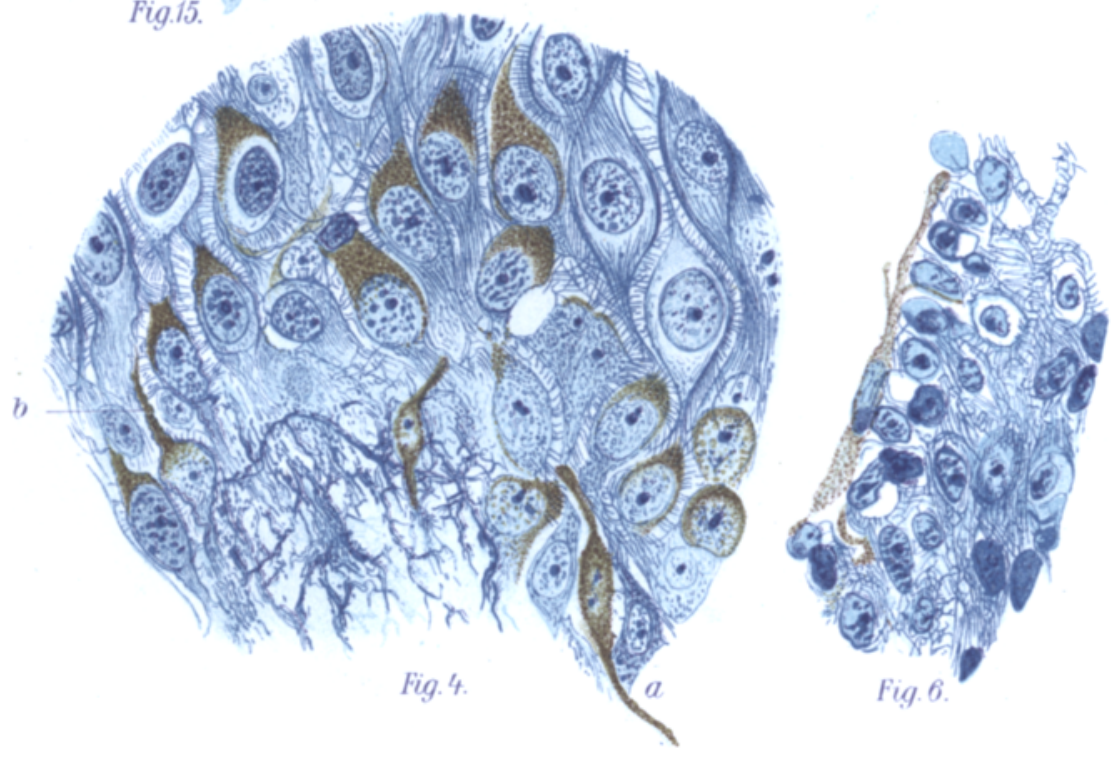

Ehrmann -0ppenheim: Über Melanoblasten. 


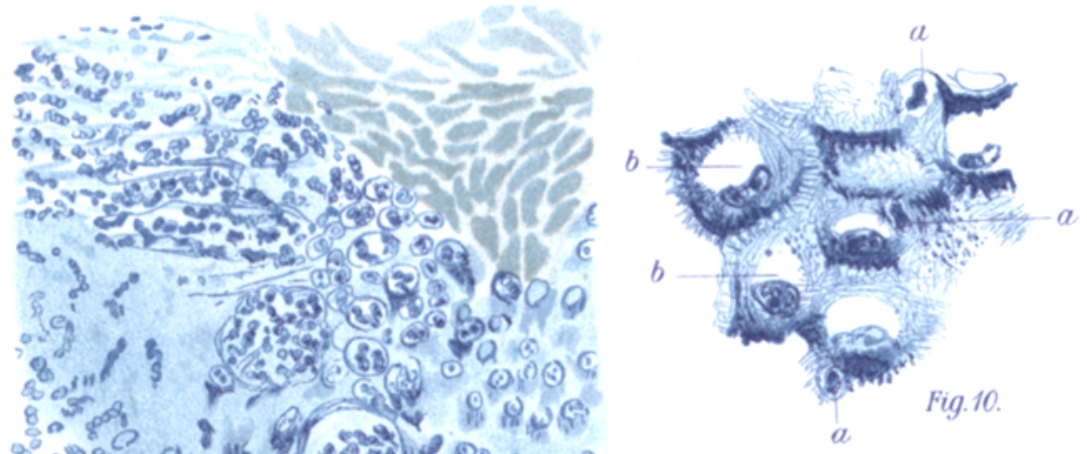

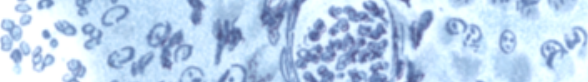

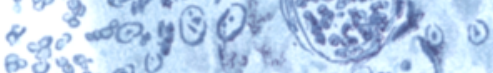

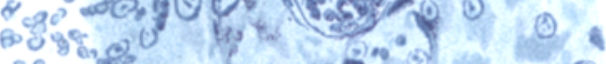

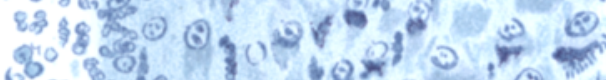

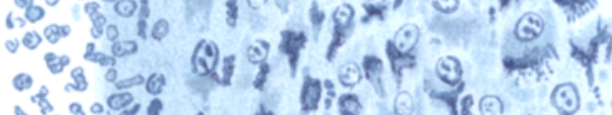

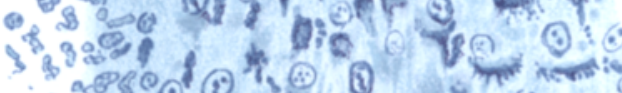

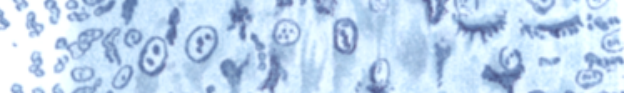

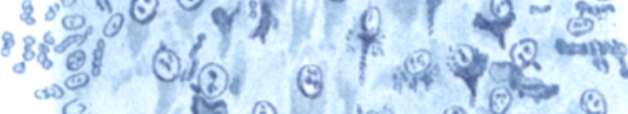

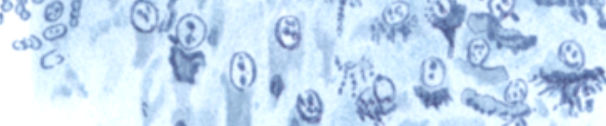

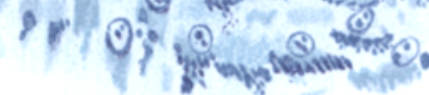

Fiq. 13

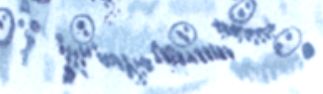

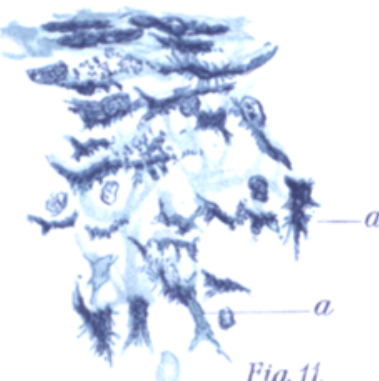

Fig. 11.
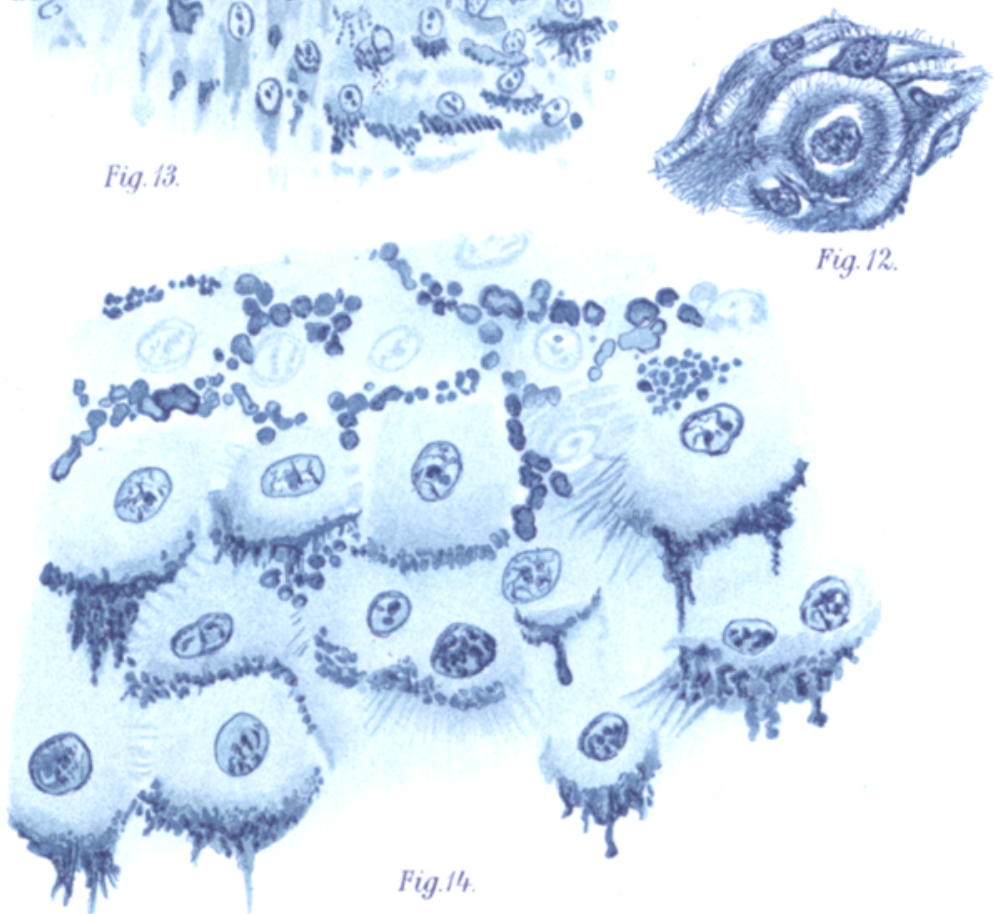